OPEN ACCESS

Edited by:

Pinyi Lu,

Frederick National Laboratory for Cancer Research (NIH), United States

Reviewed by:

Ming-Xiang Zou,

University of South China, China

Erkan Topkan

Başkent University, Turkey

*Correspondence:

Fuhua Lin

linfuh@sysucc.org.cn

Yinsheng Chen

chenyinsh@sysucc.org.cn

Jian Wang

wangjian2@sysucc.org.cn

tThese authors have contributed equally to this work

Specialty section

This article was submitted to Nutritional Immunology,

a section of the journal

Frontiers in Nutrition

Received: 20 August 2021 Accepted: 18 November 2021 Published: 17 December 2021

Citation:

Zhu S, Cheng Z, Hu Y, Chen Z Zhang J, Ke C, Yang Q, Lin F, Chen Y and Wang J (2021) Prognostic Value of the Systemic Immune-Inflammation Index and Prognostic Nutritional Index in Patients With Medulloblastoma Undergoing Surgical Resection.

Front. Nutr. 8:754958. doi: 10.3389/fnut.2021.754958

\section{Prognostic Value of the Systemic Immune-Inflammation Index and Prognostic Nutritional Index in Patients With Medulloblastoma Undergoing Surgical Resection}

\author{
Sihan Zhu ${ }^{\dagger}$, Zhuqing Cheng ${ }^{\dagger}$, Yuanjun $\mathrm{Hu}^{\dagger}$, Zhenghe Chen, Ji Zhang, Chao Ke, \\ Qunying Yang, Fuhua Lin*, Yinsheng Chen* and Jian Wang*
}

\begin{abstract}
Department of Neurosurgery and Neuro-Oncology, State Key Laboratory of Oncology in South China, Collaborative Innovation Center for Cancer Medicine, Sun Yat-sen University Cancer Center, Guangzhou, China
\end{abstract}

Background: The progression and metastasis of cancers are associated with systematic immune inflammation and nutritional dysfunction. The systemic immune-inflammation index and prognostic nutritional index (PNI) have shown a prognostic impact in several malignancies. Therefore, our study aimed to evaluate immune inflammation and nutritional index prognostic significance in patients with medulloblastoma (MB).

Methods: We retrospectively analyzed 111 patients with MB between 2001 and 2021 at our institution. The optimal cutoff values for systemic immune-inflammation index (SII), neutrophil/lymphocyte ratio (NLR), monocyte/lymphocyte counts ration (MLR), and $\mathrm{PNI}$ were evaluated with receiver operating characteristic (ROC) curve analysis. Clinical characteristics and SII, NLR, MLR, and PNI were tested with the Pearson's chi-squared test. The Kaplan-Meier survival curves and the Cox proportional hazards model were used to evaluate the effects of immune inflammation and nutritional index on overall survival (OS).

Results: Receiver operating characteristic curve analysis determined the optimal SII, NLR, MLR, and PNI cutoff values of 2,278, 14.83, 0.219, and 56.5 that significantly interacts with OS and divided the patients into two groups. Comparative survival analysis exhibited that the high-SII cohort had significantly shorter OS ( $p=0.0048$ ) than the low-SIl cohort. For the univariate analysis, the results revealed that preoperative hydrocephalus ( $p=0.01)$, SII $(p=0.006)$, albumin-bilirubin score (ALBI) $(p=0.04)$, and coSII-PNI were predictors of OS. In the multivariate analysis, preoperative hydrocephalus $(p<0.001)$, ALBI $(p=0.010)$, SII $(p<0.001)$, and coSII-PNI as independent prognostic factors were significantly correlated with OS.

Conclusion: The preoperative SII, ALBI, and coSII-PNI serve as robust prognostic biomarkers for patients with $\mathrm{MB}$ undergoing surgical resection.

Keywords: medulloblastoma, systemic immune-inflammation index, prognostic nutritional, overall survival, ALBI (albumin-bilirubin) score 


\section{INTRODUCTION}

Medulloblastoma (MB) is the most common malignant pediatric brain tumor, accounting for about $20 \%$ of central nervous system tumors of children. The current standard treatment includes surgical intervention, craniospinal irradiation, and adjuvant chemotherapy (1). The 5-year overall survival (OS) was $85 \%$ for patients with the average risk and $70 \%$ for those with the high risk (2). Sadly, this treatment has a high morbidity and mortality rate, and even those children cured are left with lifelong sequelae, including neurological, cognitive, and endocrine disorders (3). Therefore, reliable biomarkers were strongly demanded to guide treatment decisions and optimize patient outcomes.

A growing body of evidence suggested that the interaction of inflammation and the immune system with cancer cells plays a crucial role in the genesis, proliferation, progression, and metastasis of the tumors (4). Meanwhile, inflammatory pathways are considered critical targets for improving therapeutic efficacy (5). Therefore, given the relationship between inflammation and tumor development, it is of great significance to explore the application of inflammatory markers in the tumor therapy. Nutrition is essential for proper growth and development and is a critical component in optimizing clinical outcomes. Malnutrition is an adverse prognostic factor in children with cancer, and its prevalence is highly variable (6).

For the past few years, emerged novel prognostic predictive index that has been verified in many tumors. Some simple biochemical indicators can reflect systemic inflammatory response and nutritional status. Systemic immune-inflammation index (SII) was calculated by counting platelets, neutrophils, and lymphocytes in the peripheral blood. The prognostic nutritional index (PNI) was determined by serum albumin concentration and peripheral blood lymphocyte count. These two systemic immune and nutritional indicators have been shown to play a predictive role in many types of cancer (7).

Although there is compelling underlying evidence in many types of cancer, the prognostic utility of SII and PNI in patients with $\mathrm{MB}$ has, to date, surprisingly, not been carefully studied. This study focused on the prognostic role of immunoinflammatory and prognostic nutrition indicators in patients with $M B$.

\section{PATIENTS AND METHODS}

\section{Patients Section}

We retrospectively analyzed the medical data of newly diagnosed 111 patients with $\mathrm{MB}$ at our hospital from January 2001 to March 2021. Participants were in this study if they (1) were pathologically diagnosed with $\mathrm{MB}$, (2) without any antitumor treatment, (3) with complete medical follow-up data and laboratory results. The participants were excluded (1) with other primary malignant tumors found during the treatment, (2) with liver and kidney dysfunction that occurred during treatment, (3) using nonsteroidal anti-inflammatory drugs.

\section{Ethics, Consent, and Permissions}

We conducted the study per the principles of the Declaration of Helsinki and the Rules of Good Clinical Practice. Furthermore, the study design was approved by the Institutional Ethical Committee review board of Sun Yat-sen University Cancer Center (B2020-360-01) before the acquisition of any patient information. According to our institutional standards, all the patients provided a written informed consent, either by themselves or by a legally authorized representative, to collect and analyze blood samples, pathology samples, and publish their results before starting treatment.

\section{Data Collection and Definition}

The clinical parameters of patients such as gender, age, tumor size, tumor location, the Karnofsky Performance Scale (KPS), preoperative hydrocephalus, preoperative headache status, preoperative vomiting status, preoperative ataxia status, duration of symptoms, ventricular drainage status, surgery extent, metastasis status, recurrence status, postoperative radiotherapy, postoperative chemotherapy status, and laboratory data were collected from the medical data. We collected all laboratory parameters during routine tests before cancer diagnostic intervention. The ALBI grades were calculated using the following equation: $0.66 \times \log _{10}$ bilirubin level-0.085 $\times$ albumin level. The ALBI grades were stratified into grade one $(\leq-2.60)$ and grade two $(-2.59$ to -1.39$)$. The PALBI grades were calculated using the following equation: $2.02 \times \log _{10}$ bilirubin level-0.37 $\times\left(\log _{10}\right.$ bilirubin level $)-0.04 \times$ albumin level-3.48 $\times \log _{10}$ platelet count $+1.01 \times\left(\log _{10}\right.$ PLT) $(8)$. The PALBI grades were stratified into grade one $(\leq-2.53)$, grade two $(-2.52$ to -2.09$)$, and grade three (>-2.09) (8). The definitions of SII, NLR, MLR, and PNI were shown as follows: PNI $=$ albumin $(\mathrm{g} / \mathrm{L})+5 \times$ total lymphocyte counts $\left(10^{9} / \mathrm{L}\right)$; SII $=$ platelet $\times$ neutrophil/lymphocyte counts; NLR = neutrophil/lymphocyte counts; MLR = monocyte/lymphocyte counts, and PNI = albumin $(\mathrm{g} / \mathrm{L})+5 \times$ total lymphocyte counts $\left(10^{9} / \mathrm{L}\right)$. According to the existing literature reports, we will give those with low SII and high PNI score 2; those with high SII and high PNI or low SII and low PNI score 1, and those with high SII and low PNI score 0 (5). The extent of resection was recorded as gross total resection (GTR), subtotal resection (STR), and biopsy. We determined the extent of excision based on an enhanced MRI scan of the head taken within $72 \mathrm{~h}$ postoperatively and surgical records.

\section{Follow-Up}

All the postoperative patients will be regularly followed-up by full-time staffs. Follow-up work have continued until death or April 2021. OS is considered to be the time between surgery and tumor-related death or last contact.

\section{Statistical Analysis}

Software of the SPSS version 23.0 (IBM Corp, Armonk, NY) and the GraphPad Prism version 8.0 (La Jolla, CA, USA) was utilized to perform statistical analysis. Median value and range were used for the quantitative variables, while the categorical variables were described as frequency and percentage. We used the chi-squared or the Fisher's exact tests to compare groups. 
The receiver operating characteristic (ROC) curve analysis was utilized to get the optimal cutoff values of predictors by the highest Youden's index. Survival analyses were performed using the Kaplan-Meier survival curves. This study utilized Bonferroni correction and related $p$-values for comparisons between three or more groups. The Cox proportional hazards regression model investigated the univariate and multivariate analyses. Hazard ratios (HRs) and 95\% CIs were utilized to evaluate relative risk. A two-tailed $p<0.05$ was suggested remarkably significant.

\section{RESULTS}

\section{Patient Demographics}

We identified diagnosed 311 patients with MB, but 200 patients were excluded from the analysis because they only received radiotherapy or chemotherapy in our institution without surgical treatment, leaving 111 patients eligible for the following analysis. Patients with $\mathrm{MB}$ undergoing surgical resection and disease characteristics for the whole study cohort are given in Table 1. The median age was 10 (range: $1-48$ ) with male gender $(63.9 \%)$ and children with age $<18$ years $(76.5 \%)$ dominancy. The median KPS was 80 (range: 50-100). The preoperative headache, vomiting, and ataxia rates at presentation were 69.3, 64.8, and $52.2 \%$, separately. Tumor size, metastasis status, ALBI, and PALBI are also shown in Table 1.

\section{Optimal Cutoff Value for SII, NLR, MLR, and PNI}

This study applied ROC curve analysis as a more objective method to find an optimal cut-off for the possible connection between SII, NLR, MLR, PNI, and OS rather than bias-prone mean/median values. The optimal cut-off of SII, NLR, MLR, and PNI was 2,278, 14.83, 0.219, and 56.5, respectively. Details of the results are shown in Figure 1.

According to the aforementioned cut-off value, patients were divided into two groups: low-SII $(<2,278, n=102)$; high-SII $(\geq 2,278, n=9)$; low-NLR $(<14.83, n=105)$; high-NLR $(\geq$ 14.83, $n=6)$; low-MLR $(<0.219, n=57)$; high-MLR $(\geq 0.219$, $n=54)$; low-PNI ( $<56.5, n=64)$; and high-PNI $(\geq 56.5, n=$ 47). Evaluation of baseline demographics (Table 1) and treatment characteristics (Table 2) per conveyance SII, NLR, MLR, and PNI groups revealed no meaningful differences between the two cohorts, except for tumor location $(p=0.03)$ in the NLR group. Difference in gender $(p=0.03)$, age $(p=0.01)$, preoperative headache $(p=0.002)$ in the MLR group and tumor size $(p<0.01)$ in the PNI group is also found significant.

\section{Association of SII, NLR, MLR, and PNI With Survival Outcomes}

Granting the endpoints of this study, we compared the outcomes of patients allocated to the low level and high level of SII, NLR, MLR, and PNI groups in terms of OS. As shown in Figure 2, results of the comparative analyses paraded that patients with $\mathrm{MB}$ in a high level of SII had significantly inferior OS $(p=$ 0.0048 ) than patients in a low level of SII group. However, we did not find any significant difference in OS between low and high levels of patients in NLR $(p=0.49)$, MLR $(p=0.26)$, and PNI
( $p=0.38$ ) groups. Since MBs tend to occur in people younger than 18 years of age, we also made a subgroup analysis of the aforementioned immune inflammation and prognostic nutrition indexes (PNIs). Same as previous results (Figure 3), a high level of SII had significantly inferior OS $(P=0.00024)$ than patients in a low level of SII group in patients with $\mathrm{MB}$ aged $\leq 18$. Unfortunately, we have not found any significant difference in OS between low and high levels of patients in NLR $(p=0.55)$, $\operatorname{MLR}(p=0.69)$, and PNI $(p=0.44)$ groups.

\section{Univariate and Multivariate Survival Analyses}

The univariate and multivariate survival analyses are shown in Table 3. On the univariate analysis, preoperative hydrocephalus $(p=0.01)$, SII $(p=0.006)$, and ALBI $(p=0.04)$ were significantly associated with OS. Patients in the high-level SII group had an increase in death risk compared with patients in the low SII level group. Besides, the death risks of patients suffering preoperative hydrocephalus increased a lot. A high-preoperative grade of AIBL, meaning worse hepatic function, increases the risk of death in patients with $\mathrm{MB}$.

The multivariate analysis showed that preoperative hydrocephalus $(P<0.001)$, ALBI $(P=0.01)$, and SII $(p<$ $0.001)$. In terms of preoperative symptoms, patients having preoperative hydrocephalus $(\mathrm{HR}=0.047 ; 95 \%$ CI 0.012-0.185; $p$ $<0.001)$ increased the death risk. Patients in the high SII group (HR $=20.502 ; 95 \%$ CI 3.936-106.068; $p=0.01$ ) had an increase in death risk compared with patients in low SII. In addition, a higher AIBL grade $(\mathrm{HR}=6.915 ; 95 \%$ CI $1.587-19.253 ; p=0.01)$ can significantly increase a risk of death of patient.

\section{Prognostic Value of the coSII-PNI in MB Undergoing Surgical Resection}

Finally, we evaluated the prognostic value of the coSII-PNI in patients with $\mathrm{MB}$ undergoing surgical resection. Those with low SII and high PNI will be given a score of two; those with high SII and high PNI or low SII or low PNI score one; those with high SII and low PNI score 0. The Kaplan-Meier analysis and a log-rank test for the entire patient cohort showed that the 5-year OS for patients with $\operatorname{coSII}-\mathrm{PNI}=0,1$, and 2 were $0,16.5$, and $37.5 \%$, respectively. Therefore, patients with $\mathrm{MB}$ were divided into three independent groups by preoperative coSII-PNI ( $p$ $=0.006$ ) (Figure 4). Subgroup analysis was then performed to evaluate the prognostic value of coSII-PNI for age stratification. Under the age of 18 , the highest prevalence of $\mathrm{MB}$, we found coSII-PNI $=0$ was inclined to have a worse OS than patients with coSII-PNI $=1$ or coSII-PNI $=2(p=0.024)$ (Figure 4). However, no significant difference was found in adult patients $(p=0.57)$. The univariate and multivariate analyses also proved coSII-PNI was an independent prognostic factor in patients with MB (Table 3).

\section{DISCUSSION}

According to our best information, this study is the first attempt to specifically question the prognostic effect of the 
TABLE 1 | Baseline patient and disease characteristics.

\begin{tabular}{|c|c|c|c|c|c|c|c|c|c|c|c|c|c|}
\hline \multirow[b]{3}{*}{ Variables } & \multicolumn{4}{|c|}{ SII } & \multicolumn{2}{|c|}{ NLR } & & \multicolumn{2}{|c|}{ MLR } & \multicolumn{4}{|c|}{ PNI } \\
\hline & & Low & High & & Low & High & & Low & High & & Low & High & \\
\hline & Cases & (102) & (9) & $P$ & (105) & (6) & $P$ & (57) & (54) & $P$ & (64) & (47) & $P$ \\
\hline Gender & & & & 0.58 & & & 0.88 & & & 0.03 & & & 0.43 \\
\hline Male & 71 & 66 & 5 & & 67 & 4 & & 31 & 40 & & 39 & 32 & \\
\hline Female & 40 & 36 & 4 & & 38 & 2 & & 26 & 14 & & 25 & 15 & \\
\hline Age(years) & & & & 0.46 & & & 0.68 & & & 0.01 & & & 0.36 \\
\hline$<18$ & 85 & 79 & 6 & & 80 & 5 & & 49 & 36 & & 47 & 38 & \\
\hline$\geq 18$ & 26 & 23 & 3 & & 25 & 1 & & 8 & 18 & & 17 & 9 & \\
\hline Tumor size & & & & 0.64 & & & 0.80 & & & 0.54 & & & $<0.01$ \\
\hline$\leq 4 \mathrm{~cm}$ & 32 & 30 & 2 & & 30 & 2 & & 15 & 17 & & 27 & 5 & \\
\hline$>4 \mathrm{~cm}$ & 79 & 72 & 7 & & 75 & 4 & & 42 & 37 & & 37 & 42 & \\
\hline Tumor location & & & & 0.60 & & & 0.03 & & & 0.07 & & & 0.13 \\
\hline Subtentorial & 108 & 99 & 9 & & 103 & 5 & & 57 & 51 & & 61 & 47 & \\
\hline Subtentorial-supracerebellar & 3 & 3 & 0 & & 2 & 1 & & 0 & 3 & & 3 & 0 & \\
\hline KPS & & & & 0.50 & & & 0.96 & & & 0.16 & & & 0.71 \\
\hline$\leq 70$ & 38 & 34 & 4 & & 36 & 2 & & 16 & 22 & & 21 & 17 & \\
\hline$>70$ & 73 & 68 & 5 & & 69 & 4 & & 41 & 32 & & 43 & 30 & \\
\hline Preoperative hydrocephalus & & & & 0.28 & & & 0.49 & & & 0.54 & & & 0.53 \\
\hline Yes & 79 & 74 & 5 & & 74 & 5 & & 42 & 37 & & 47 & 32 & \\
\hline No & 32 & 28 & 4 & & 31 & 1 & & 15 & 17 & & 17 & 15 & \\
\hline Preoperative headache & & & & 0.18 & & & 0.09 & & & 0.002 & & & 0.055 \\
\hline Yes & 77 & 69 & 8 & & 71 & 6 & & 32 & 45 & & 49 & 28 & \\
\hline No & 34 & 33 & 1 & & 34 & 0 & & 25 & 9 & & 15 & 19 & \\
\hline Preoperative vomiting & & & & 0.90 & & & 0.92 & & & 0.99 & & & 0.84 \\
\hline Yes & 72 & 66 & 6 & & 68 & 4 & & 37 & 35 & & 42 & 30 & \\
\hline No & 39 & 36 & 3 & & 37 & 2 & & 20 & 19 & & 22 & 17 & \\
\hline Preoperative ataxia & & & & 0.83 & & & 0.91 & & & 0.93 & & & 0.86 \\
\hline Yes & 58 & 53 & 5 & & 55 & 3 & & 30 & 28 & & 33 & 25 & \\
\hline No & 53 & 49 & 4 & & 50 & 3 & & 27 & 26 & & 31 & 22 & \\
\hline Duration of symptoms & & & & 0.05 & & & 0.12 & & & 0.86 & & & 0.32 \\
\hline$\leq 3$ months & 81 & 72 & 9 & & 75 & 6 & & 42 & 39 & & 49 & 32 & \\
\hline$>3$ months & 30 & 30 & 9 & & 30 & 0 & & 15 & 15 & & 15 & 15 & \\
\hline Metastasis & & & & 0.45 & & & 0.80 & & & 0.07 & & & 0.07 \\
\hline Yes & 23 & 22 & 1 & & 22 & 1 & & 8 & 15 & & 17 & 6 & \\
\hline No & 88 & 80 & 8 & & 83 & 5 & & 49 & 39 & & 47 & 41 & \\
\hline ALBI & & & & 0.41 & & & 0.51 & & & 0.75 & & & 0.44 \\
\hline Grade 1 & 104 & 95 & 9 & & 98 & 6 & & 53 & 51 & & 59 & 45 & \\
\hline Grade 2 & 7 & 7 & 0 & & 7 & 0 & & 4 & 3 & & 5 & 2 & \\
\hline PALBI & & & & 0.14 & & & 0.83 & & & 0.10 & & & 0.37 \\
\hline Grade 1 & 83 & 75 & 8 & & 78 & 5 & & 46 & 37 & & 48 & 35 & \\
\hline Grade 2 & 24 & 24 & 0 & & 23 & 1 & & 8 & 16 & & 15 & 9 & \\
\hline Grade 3 & 4 & 3 & 1 & & 4 & 0 & & 3 & 1 & & 1 & 3 & \\
\hline Mean OS time (month) & & 45.1 & 36.3 & & 44.7 & 39.1 & & 35.9 & 32.5 & & 33.1 & 52.8 & \\
\hline
\end{tabular}

SII, systemic immune-inflammation index; NLR, neutrophil-lymphocyte ratio; MLR, monocyte-lymphocyte ratio; PNI, prognostic nutritional index.

immunoinflammatory index and nutritional index on survival outcomes in newly diagnosed patients with $\mathrm{MB}$ undergoing surgical resection. We demonstrated that the preoperative highlevel SII was linked with significantly inferior OS than lowlevel SII in this group of patients. Moreover, we proved that a high level of SII and higher score ALBI increased the risk of death in patients with MB. Furthermore, we introduced a new concept called coSII-PNI to predict OS in patients with MB. Therefore, in addition to recognized clinicopathological factors, namely, preoperative hydrocephalus, our results suggested a 


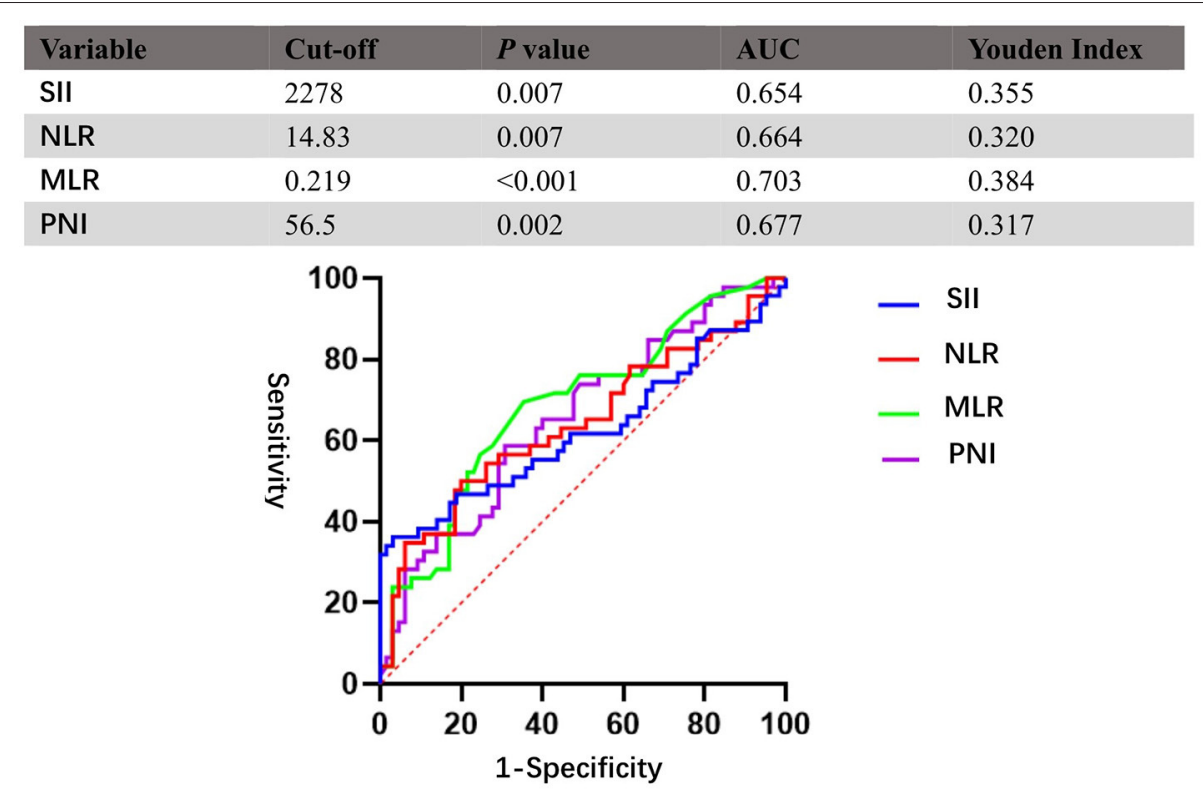

FIGURE 1 | Receiver operating characteristic curves (ROCs) for pretreatment SII, NLR, MLR, and PNI based on OS. SII, systemic immune-inflammation index; NLR, neutrophil-lymphocyte ratio; MLR, monocyte-lymphocyte ratio; PNI, prognostic nutritional index.

TABLE 2 | Treatment characteristics and clinical outcomes.

\begin{tabular}{|c|c|c|c|c|c|c|c|c|c|c|c|c|c|}
\hline \multirow[b]{3}{*}{ Variables } & & \multicolumn{2}{|c|}{ SII } & \multicolumn{4}{|c|}{ NLR } & \multicolumn{2}{|c|}{ MLR } & \multicolumn{4}{|c|}{ PNI } \\
\hline & & Low & High & & Low & High & & Low & High & & Low & High & \\
\hline & Cases & (102) & (9) & $\boldsymbol{P}$ & (105) & (6) & $\boldsymbol{P}$ & (57) & (54) & $\boldsymbol{P}$ & (64) & (47) & $P$ \\
\hline Postoperative drainage & & & & 0.15 & & & 0.08 & & & 0.53 & & & 0.08 \\
\hline Yes & 75 & 67 & 8 & & 69 & 6 & & 37 & 38 & & 39 & 36 & \\
\hline No & 36 & 35 & 1 & & 36 & 0 & & 20 & 16 & & 25 & 11 & \\
\hline Recurrence & & & & 0.78 & & & 0.13 & & & 0.96 & & & 0.10 \\
\hline Yes & 29 & 2 & 6 & & 29 & 0 & & 15 & 14 & & 13 & 16 & \\
\hline No & 82 & 75 & 7 & & 76 & 7 & & 42 & 40 & & 51 & 31 & \\
\hline Surgical extent & & & & 0.84 & & & 0.78 & & & 0.29 & & & 0.42 \\
\hline GTR & 79 & 73 & 6 & & 74 & 5 & & 40 & 39 & & 46 & 33 & \\
\hline STR & 30 & 27 & 3 & & 29 & 1 & & 17 & 13 & & 16 & 14 & \\
\hline Biopsy & 2 & 0 & 2 & & 2 & 0 & & 0 & 2 & & & & \\
\hline Postoperative radiotherapy & & & & 0.53 & & & 0.16 & & & 0.31 & & & 0.59 \\
\hline Yes & 67 & 62 & 5 & & 65 & 2 & & 37 & 30 & & 40 & 27 & \\
\hline No & 44 & 40 & 4 & & 40 & 4 & & 20 & 24 & & 24 & 20 & \\
\hline Postoperative chemotherapy & & & & 0.53 & & & 0.73 & & & 0.84 & & & 0.90 \\
\hline Yes & 63 & 57 & 6 & & 60 & 3 & & 33 & 30 & & 36 & 27 & \\
\hline No & 48 & 45 & 3 & & 45 & 3 & & 24 & 24 & & 28 & 20 & \\
\hline
\end{tabular}

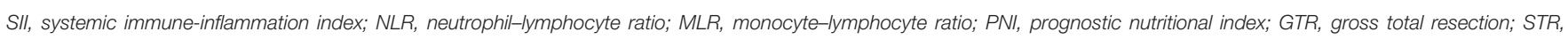
subtotal resection.

reliable and independent role of a new inexpensive and clinically relevant biomarkers SII, AIBL, and coSII-PNI for future prognosis prediction.

Several prognostic scores based on the inflammation and immunity have been developed to identify patients at high risk of relapse or death that may be difficult to identify using traditional clinicopathological indicators. However, these scores do not fully reflect the balance of host inflammatory and immune status. Therefore, we established a new indicator based on lymphocyte, neutrophil, and platelet counts, the SII index. Baseline SII can be used as an independent prognostic marker in patients with advanced pancreatic cancer with normal or 

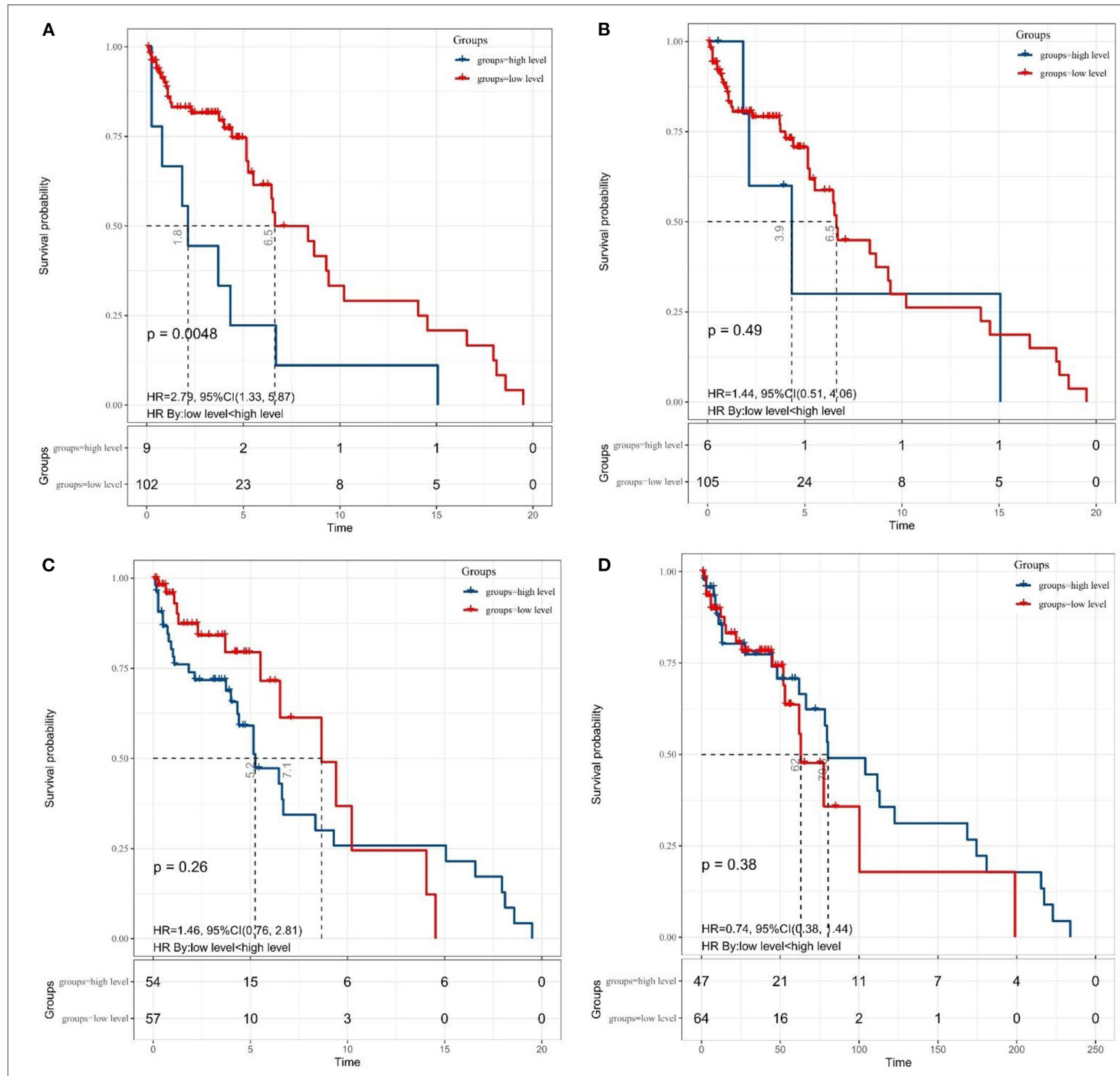

FIGURE 2 | The Kaplan-Meier survival curves for OS according to (A) SII, (B) NLR, (C) MLR, and (D) PNI in patients with medulloblastoma. SII, systemic immune-inflammation index; NLR, neutrophil-lymphocyte ratio; MLR, monocyte-lymphocyte ratio; PNI, prognostic nutritional index.

elevated CA19-9 levels (9). Data obtained from the Rotterdam Study showed that higher SII at baseline was associated with a $30 \%$ increased risk of solid cancer, adjusted for age, sex, socioeconomic status, smoking status, body mass index, and type two diabetes. Absolute cumulative 10-year cancer risk increased from $9.7 \%$ in the lowest quartile of SII to $14.7 \%$ in the highest quartile of SII ( $p=0.009$ ) (10). The PNI is a new prognostic score calculated by multiplying albumin and lymphocyte counts to reflect inflammation and nutritional status (11). A high decrease in PNI during neoadjuvant chemotherapy predicts a poor prognosis. Maintaining nutritional status during neoadjuvant chemotherapy might lead to better treatment outcomes for the patients with breast cancer (12). Therefore, SII and PNI are prognostic indicators worthy of investigation in $\mathrm{MB}$.

Studies have shown that the tumor progression, immunity, and inflammatory response have a special relationship. As a novel finding in the modern $\mathrm{MB}$ literatures, this study convincingly demonstrated that high levels of SII were strongly and independently associated with more inferior OS of patients with $\mathrm{MB}$ undergoing surgical resection. Albeit this is the first 

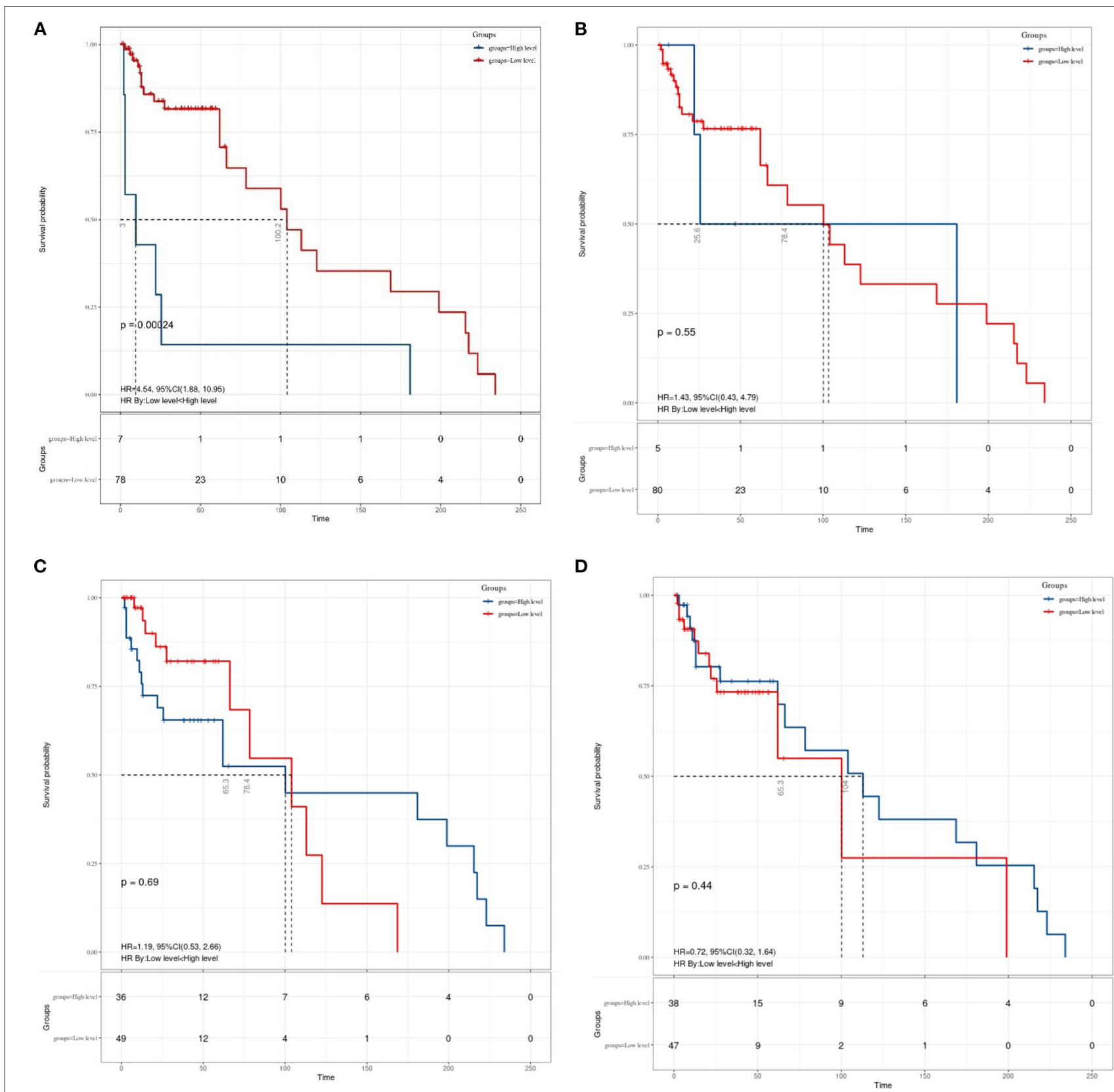

FIGURE 3 | The Kaplan-Meier survival curves for OS according to (A) SII, (B) NLR, (C) MLR, and (D) PNI in patients aged $\leq 18$ years with medulloblastoma. SII, systemic immune-inflammation index; NLR, neutrophil-lymphocyte ratio; MLR, monocyte-lymphocyte ratio; PNI, prognostic nutritional index.

report to show an essential association between SII and the survival outcome of patients with $\mathrm{MB}$, they are harmonious with previous research on other tumors of central nervous system (13-15). Therefore, a better understanding of the role of these biomarkers in $\mathrm{MB}$ will help explore the relationship between inflammation, immunity, and $\mathrm{MB}$. However, the underlying mechanisms require further evaluation.

Patients with $\mathrm{MB}$ with high SII usually have elevated neutrophils, high platelets, or lymphocytopenia, indicating increased inflammation and reduced immune response. Neutrophils have been reported to be involved in the tumor formation and progression through various mechanisms, including vascular endothelial growth factor-mediated angiogenesis and tumor immunosuppression (16). Study of Karajannis et al. already proved patients with MB have a higher neutrophil count suggesting patients already had tumor-induced systemic immunosuppression at the time of diagnosis (17). On the other hand, platelets play an active role in all steps of tumorigenesis, including tumor growth, tumor cell exosmosis, and metastasis. In addition, thrombocytosis in patients with cancer is associated with poor survival. Due to many particles and exosomes secreted, platelets can well coordinate local 
TABLE 3 | The univariate and multivariate analyses of overall survival in patients with medulloblastoma.

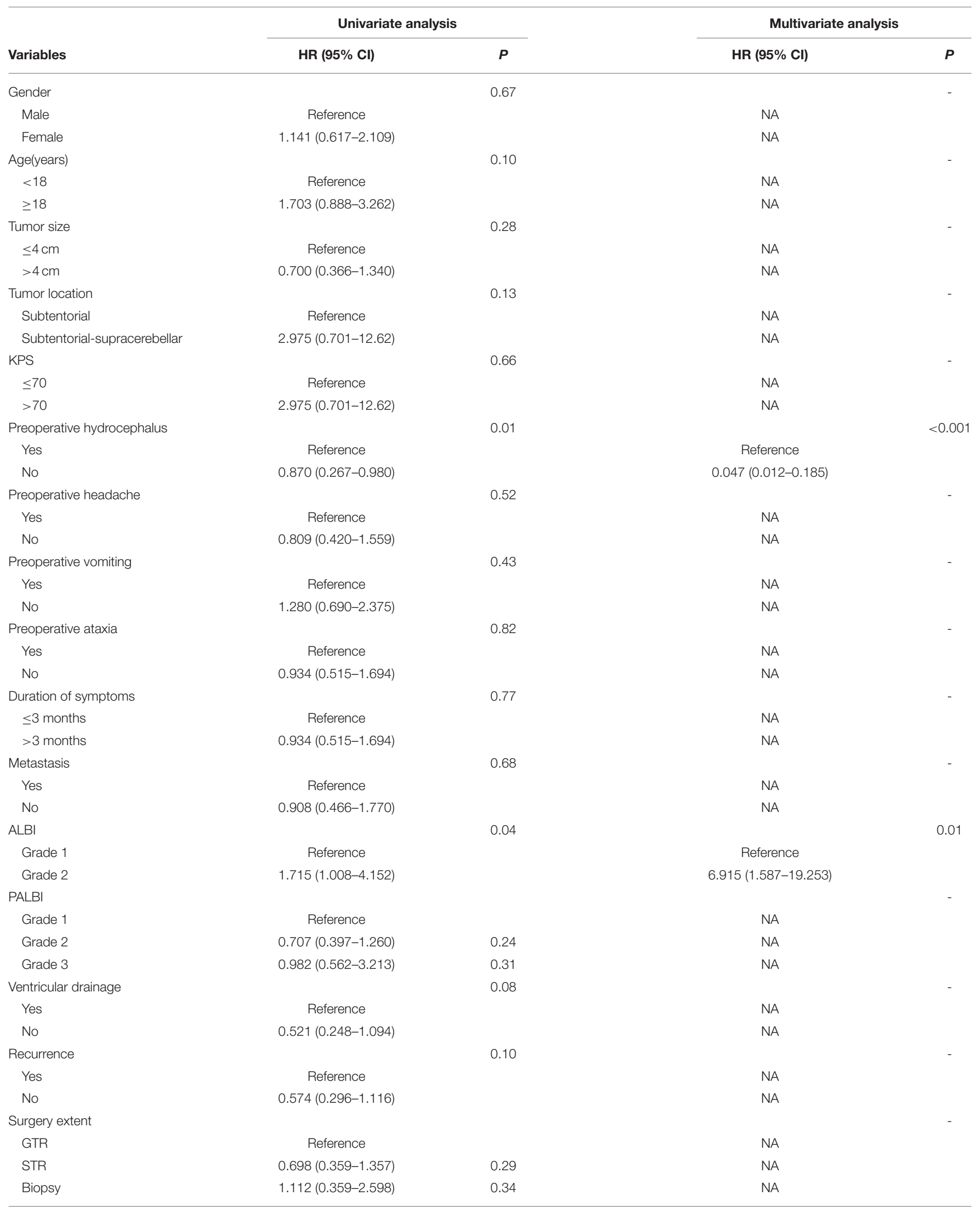


TABLE 3 | Continued

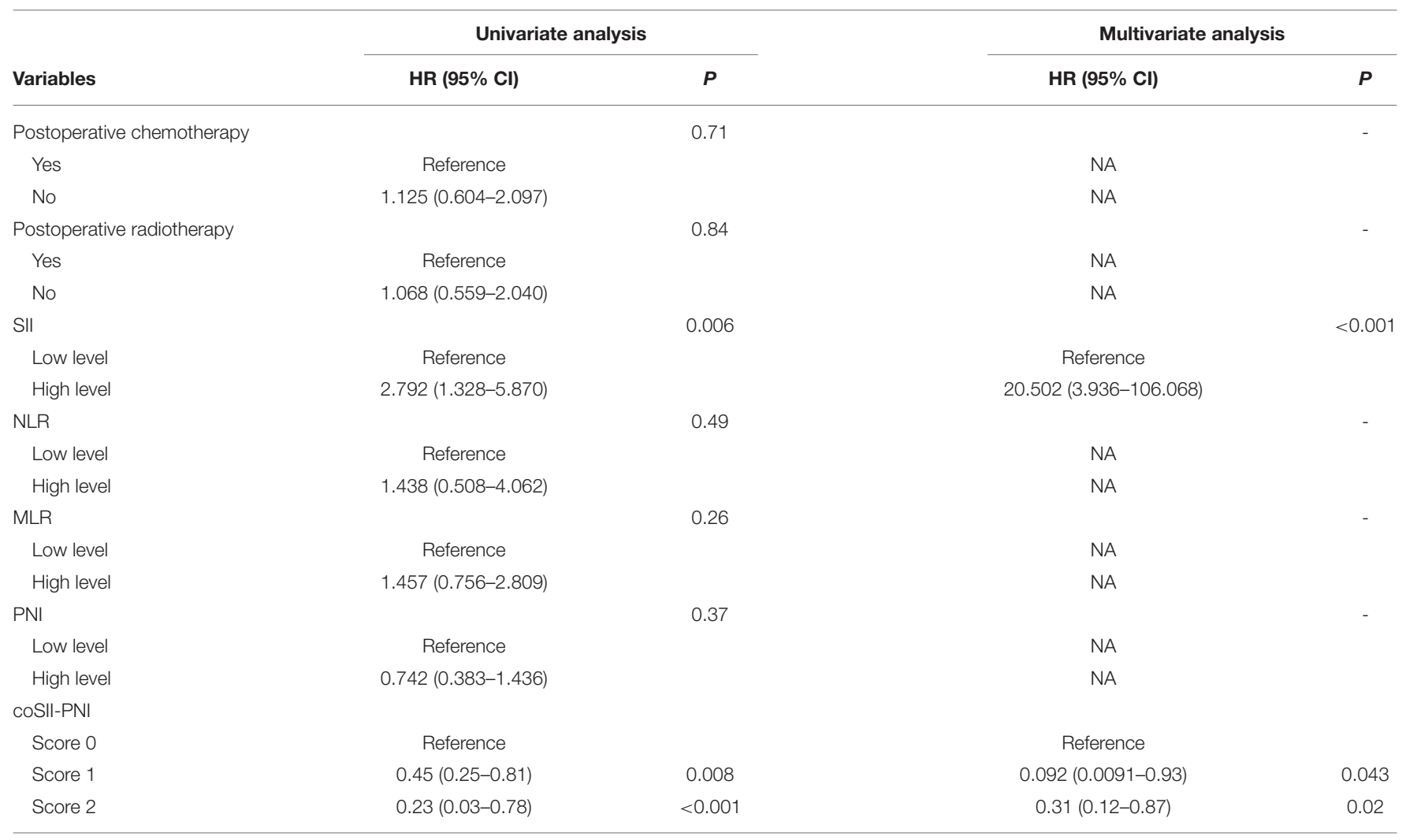

SII, systemic immune-inflammation index; NLR, neutrophil-lymphocyte ratio; MLR, monocyte-lymphocyte ratio; PNI, prognostic nutritional index; HR, hazard ratio.

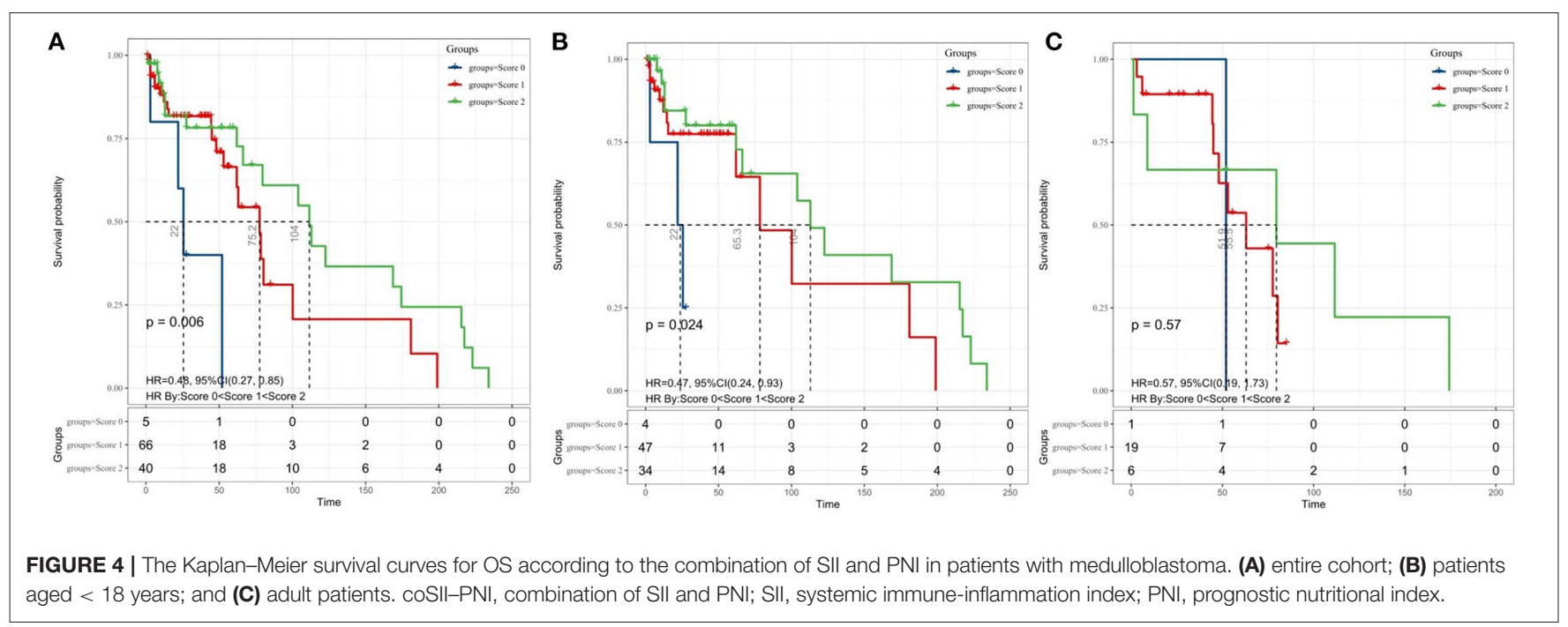

and distant tumor-host crosstalk (18). Expression of plateletderived growth factors was also common in gliomas (19). Hambardzumyan et al. study demonstrated platelet-derived growth factor-beta is a potent inflammatory driver in pediatric MB (19). Lymphocytes reflect host immune status and mediate host immune response to cancer. Meanwhile, lymphocytes have an antitumor effect by inhibiting proliferation and invasion of cancer cells and promoting apoptosis of tumor cells. Tumor-infiltrating lymphocytes play an essential role in mediating responses to chemotherapy and improving cancer clinical outcomes (20). What is more, systemic inflammation may increase neutrophils count and decrease lymphocyte count, which may lead to a reduction in cell-mediated cytotoxic immune response, leading to treatment failure (21). To confirm the difference of the inflammatory and the immune response status in the molecular subgroups, Zhang et al. showed that 
the preoperative levels of NLR in the group three MB were significantly higher than those in the WNT group, and the preoperative high NLR was negatively correlated with OS in group three $(p=0.032)$ and group four $(p=0.027)$ (22). Therefore, anti-inflammatory therapy or immunotherapy may be an effective treatment strategy for patients with MB with high SII. These studies may help shed light on why patients with high SII have worse outcomes and suggest the potential for anti-inflammatory therapy in patients with $\mathrm{MB}$, although further research is needed to verify this hypothesis.

Index has been extensively studied in other CNS tumors. The recent studies suggest that NLR may be a novel biomarker for the prognostic stratification of R-GBMS treated with BEVIRI (23). Another study conducted by Dr. Erkan Topkan (24) showed that preconditioning pretreatment systemic immune response index (SIRI) is a new, reliable, and independent predictor of prognosis for newly diagnosed patients with GBM intending to undergo postoperative Stupp regiments. As a tumor of the central nervous system, the prognostic prediction of $\mathrm{MB}$ by the cerebrospinal fluid (CSF) is progressing rapidly. For the common tumor markers seen in the blood, the CSF tends to show a different performance. This study found that circulating tumor DNA (ctDNA) is abundant in CSF but almost absent in plasma, and longitudinal analysis of ctDNA in CSF is useful in studying the characteristics of minimal residual disease, genomic evolution, and the recurrent tumors in $\mathrm{MB}$ (25). However, no prognostic value was found for immunoinflammatory markers in CSF. In fact, researchers have found that proteomic analysis identified typical tumor markers, including FSTL5, ART3, and FMOD, and revealed the prevalence of anti-inflammatory and tumor-promoting proteins that are characteristic of myeloid cells in CSF from patients with MB (26). This also provides some theoretical basis for the application of the immune inflammation index in $\mathrm{MB}$ in the future.

Albumin-Bilirubin score, a well-designed marker composed of bilirubin and albumin, can be used as an assessment tool for hepatic function. Albumin can be a multifunctional protein carrier system for tumor therapy. As a carrier, it can provide tumor specificity, reduce drug-related toxicity, and maintain the therapeutic concentration of active parts such as drugs, genes, peptides, and proteins (27). Albumin nanoparticles for glutathione-responsive release of cisplatin can be used in the treatment of $\mathrm{MB}$ and this dramatically increases the efficiency of cisplatin crossing the blood-brain barrier (28). Bilirubin, a by-product of the breakdown of hemoglobin, is said to be an antioxidant and is thought to protect against cancer (29). Moderately elevated serum bilirubin levels are associated with a lower incidence of lung cancer, especially among smokers. It is not clear whether these relationships reflect antioxidant properties or residual confusion of cancer (30). As a nutritional index to evaluate liver function, it is often used to evaluate hepatocellular carcinoma (HCC) tolerance of patients to treatment (31). A higher ALBI grade predicted a poorer prognosis for HCC (32). Our study was the first one in tumors other than patients with HCC to illustrate significant connections between the ALBI grade and survival outcomes. The specific mechanism of the liver metabolic nutrition and prognosis of $\mathrm{MB}$ deserves further investigation.

Prognostic nutritional index is considered to be a potent negative prognostic factor in many cancers $(33,34)$. However, no previous studies had characterized prognostic value of PNI in MB. Similarly, PNI was not an independent prognostic factor in our study. We considered that the deviation might be caused by insufficient sample size and retrospective study. Besides, the findings suggested that early aggressive nutritional interventions need to be considered to prevent nutritional decline during $\mathrm{MB}$ treatment (35). Therefore, we utilized a combinative analysis of SII and PNI to predict the OS of patients with MB. Our data showed that patients with high PNI and low SII had the best prognosis, while patients with low PNI and high SII had the worst prognosis. In age $<18$ years patients who are high-risk groups of $\mathrm{MB}$, coSII-PNI was significantly correlated with OS $(P=0.0024)$. All the aforementioned results suggest that we cannot ignore the predictive role of PNI in MB, which needs to be validated in large prospective clinical trials.

This study has some certain hindrances. First, this is a single-institution retrospective cohort analysis in a relatively small cohort. Therefore, our findings should be interpreted with considerable caution until consistent results of prospective hypotheses with the large-scale confirmatory studies are possible. Second, tumor-related variables such as molecular subgroups and local/systemic reactive proinflammatory cytokine/chemokine levels did not allow us to perform SII group analysis based on these biomarkers. Third, several reported evaluations of inflammatory markers, including C-reactive protein, Glasgow prognostic score, interleukin, and tumor necrosis factor, were not included in this study (36). Last but not the least, unforeseeable differences between the salvage maneuvers at recurrence may have altered the outcomes in favor of one group in an unintentional manner. Therefore, more extensive prospective studies, especially confirmatory studies and Hematological indexes measured at multiple times, are needed in the future to confirm our preliminary results.

\section{CONCLUSION}

The present endeavor exploring the prognostic significance of immune-nutritional index on survival results of the patients with $\mathrm{MB}$ undergoing surgical resection setting exhibited that the reproducibly measurable, cost-effective, and easily calculated SII, AIBL, and coSII-PNI were predictors of OS in patients with $\mathrm{MB}$. If consistent with the results of future large-scale studies, we rationally believe that future well-designed studies addressing these questions may provide valuable insights into the mechanistic relationship of these biomarkers in patients with MB.

\section{DATA AVAILABILITY STATEMENT}

The original contributions presented in the study are included in the article/supplementary material, further inquiries can be directed to the corresponding authors. 


\section{ETHICS STATEMENT}

The studies involving human participants were reviewed and approved by Institutional Review Board of Sun Yat-sen University Cancer Center. Written informed consent to participate in this study was provided by the participants' legal guardian/next of kin.

\section{REFERENCES}

1. Liu X, Ding C, Tan W, Zhang A. Medulloblastoma: molecular understanding, treatment evolution, and new developments. Pharmacol Ther. (2020) 210:107516. doi: 10.1016/j.pharmthera.2020.107516

2. Tarbell NJ, Friedman H, Polkinghorn WR, Yock T, Zhou T, Chen Z, et al. High-risk medulloblastoma: a pediatric oncology group randomized trial of chemotherapy before or after radiation therapy (POG 9031). J Clin Oncol. (2013) 31:2936-41. doi: 10.1200/JCO.2012.43.9984

3. Mulhern RK, Reddick WE, Palmer SL, Glass JO, Elkin TD, Kun LE, et al. Neurocognitive deficits in medulloblastoma survivors and white matter loss. Ann Neurol. (1999) 46:834-41.

4. Aggarwal BB, Vijayalekshmi RV, Sung B. Targeting inflammatory pathways for prevention and therapy of cancer: short-term friend, long-term foe. Clin Cancer Res. (2009) 15:425-30. doi: 10.1158/1078-0432.CCR-08-0149

5. Zhang H, Shang X, Ren P, Gong L, Ahmed A, Ma Z, et al. The predictive value of a preoperative systemic immune-inflammation index and prognostic nutritional index in patients with esophageal squamous cell carcinoma. J Cell Physiol. (2019) 234:1794-802. doi: 10.1002/jcp.27052

6. Viani K, Trehan A, Manzoli B, Schoeman J. Assessment of nutritional status in children with cancer: a narrative review. Pediatr Blood Cancer. (2020) 67 Suppl 3:e28211. doi: 10.1002/pbc.28211

7. Topkan E, Besen AA, Ozdemir Y, Kucuk A, Mertsoylu H, Pehlivan B, et al. Prognostic value of pretreatment systemic immune-inflammation index in glioblastoma multiforme patients undergoing postneurosurgical radiotherapy plus concurrent and adjuvant temozolomide. Mediators Inflamm. (2020) 2020:4392189. doi: 10.1155/2020/4392189

8. Lu LH, Zhang YF, Mu-Yan C, Kan A, Zhong XP, Mei J, et al. Plateletalbumin-bilirubin grade: risk stratification of liver failure, prognosis after resection for hepatocellular carcinoma. Dig Liver Dis. (2019) 51:14307. doi: 10.1016/j.dld.2019.04.006

9. Zhang K, Hua YQ, Wang D, Chen LY, Wu CJ, Chen Z, et al. Systemic immuneinflammation index predicts prognosis of patients with advanced pancreatic cancer. J Transl Med. (2019) 17:30. doi: 10.1186/s12967-019-1782-x

10. Fest J, Ruiter R, Mulder M, Groot Koerkamp B, Ikram MA, Stricker BH, et al. The systemic immune-inflammation index is associated with an increased risk of incident cancer-A population-based cohort study. Int J Cancer. (2020) 146:692-8. doi: 10.1002/ijc.32303

11. Mirili C, Yilmaz A, Demirkan S, Bilici M, Basol Tekin S. Clinical significance of prognostic nutritional index (PNI) in malignant melanoma. Int J Clin Oncol. (2019) 24:1301-10. doi: 10.1007/s10147-019-01461-7

12. Oba T, Maeno K, Takekoshi D, Ono M, Ito T, Kanai T, et al. Neoadjuvant chemotherapy-induced decrease of prognostic nutrition index predicts poor prognosis in patients with breast cancer. BMC Cancer. (2020) 20:160. doi: 10.1186/s12885-020-6647-4

13. Liang $\mathrm{R}$, Li J, Tang $\mathrm{X}$, Liu $\mathrm{Y}$. The prognostic role of preoperative systemic immune-inflammation index and albumin/globulin ratio in patients with newly diagnosed high-grade glioma. Clin Neurol Neurosurg. (2019) 184:105397. doi: 10.1016/j.clineuro.2019.105397

14. Xu W, Wang D, Zheng X, Ou Q, Huang L. Sex-dependent association of preoperative hematologic markers with glioma grade and progression. $J$ Neurooncol. (2018) 137:279-87. doi: 10.1007/s11060-017-2714-3

15. Li M, Bai J, Wang S, Zhai Y, Zhang S, Li C, et al. Clinical implication of systemic immune-inflammation index and prognostic nutritional index in skull base chordoma patients. Front Oncol. (2021) 11:548325. doi: 10.3389/fonc.2021.548325

\section{AUTHOR CONTRIBUTIONS}

SZ and ZCheng conducted data analysis and drafted the manuscript. YH, ZChen, and JZ participated in the data collection. JW, YC, and FL participated in the design of the study. All authors read and approved the final manuscript.

16. Liang W, Ferrara N. The complex role of neutrophils in tumor angiogenesis and metastasis. Cancer Immunol Res. (2016) 4:83-91. doi: 10.1158/2326-6066.CIR-15-0313

17. Patel S, Wang S, Snuderl M, Karajannis MA. Pre-treatment lymphopenia and indication of tumor-induced systemic immunosuppression in medulloblastoma. J Neurooncol. (2018) 136:541-4. doi: 10.1007/s11060-017-2678-3

18. Haemmerle M, Stone RL, Menter DG, Afshar-Kharghan V, Sood AK. The platelet lifeline to cancer: challenges and opportunities. Cancer Cell. (2018) 33:965-83. doi: 10.1016/j.ccell.2018.03.002

19. Ross JL, Chen Z, Herting CJ, Grabovska Y, Szulzewsky F, Puigdelloses $\mathrm{M}$, et al. Platelet-derived growth factor beta is a potent inflammatory driver in paediatric high-grade glioma. Brain. (2021) 144:53-69. doi: 10.1093/brain/awaa382

20. Stanton SE, Disis ML. Clinical significance of tumor-infiltrating lymphocytes in breast cancer. J Immunother Cancer. (2016) 4:59. doi: 10.1186/s40425-016-0165-6

21. Bambury RM, Teo MY, Power DG, Yusuf A, Murray S, Battley JE, et al. The association of pre-treatment neutrophil to lymphocyte ratio with overall survival in patients with glioblastoma multiforme. J Neurooncol. (2013) 114:149-54. doi: 10.1007/s11060-013-1164-9

22. Li K, Duan WC, Zhao HB, Wang L, Wang WW, Zhan YB, et al. Preoperative neutrophil to lymphocyte ratio and platelet to lymphocyte ratio are associated with the prognosis of group 3 and group 4 medulloblastoma. Sci Rep. (2019) 9:13239. doi: 10.1038/s41598-019-49733-6

23. Haksoyler V. A Besen A, Koseci T, Olgun P, Bayram E, Topkan E. Neutrophilto-lymphocyte ratio is prognostic in recurrent glioblastoma multiforme treated with bevacizumab plus irinotecan. Biomark Med. (2021) 15:8519. doi: 10.2217/bmm-2021-0271

24. Topkan E, Kucuk A, Ozdemir Y, Mertsoylu H, Besen AA, Sezen D, et al. Systemic inflammation response index predicts survival outcomes in glioblastoma multiforme patients treated with standard stupp protocol. $J$ Immunol Res. (2020) 2020:8628540. doi: 10.1155/2020/8628540

25. Escudero L, Llort A, Arias A, Diaz-Navarro A, Martínez-Ricarte F, RubioPerez C, et al. Circulating tumour DNA from the cerebrospinal fluid allows the characterisation and monitoring of medulloblastoma. Nat Commun. (2020) 11:5376. doi: 10.1038/s41467-020-19175-0

26. Hu B, Yang XR, Xu Y, Sun YF, Sun C, Guo W, et al. Systemic immune-inflammation index predicts prognosis of patients after curative resection for hepatocellular carcinoma. Clin Cancer Res. (2014) 20:621222. doi: 10.1158/1078-0432.CCR-14-0442

27. Kudarha RR, Sawant KK. Albumin based versatile multifunctional nanocarriers for cancer therapy: fabrication, surface modification, multimodal therapeutics and imaging approaches. Mater Sci Eng C Mater Biol Appl. (2017) 81:607-26. doi: 10.1016/j.msec.2017.08.004

28. Catanzaro G, Curcio M, Cirillo G, Spizzirri UG, Besharat ZM, Abballe L, et al. Albumin nanoparticles for glutathione-responsive release of cisplatin: new opportunities for medulloblastoma. Int J Pharm. (2017) 517:16874. doi: 10.1016/j.ijpharm.2016.12.017

29. Seyed Khoei N, Jenab M, Murphy N, Banbury BL, Carreras-Torres R, Viallon V, et al. Circulating bilirubin levels and risk of colorectal cancer: serological and Mendelian randomization analyses. BMC Med. (2020) 18:229. doi: 10.1186/s12916-020-01703-w

30. Horsfall LJ, Burgess S, Hall I, Nazareth I. Genetically raised serum bilirubin levels and lung cancer: a cohort study and Mendelian randomisation using UK Biobank. Thorax. (2020) 75:955-64. doi: 10.1136/thoraxjnl-2020-214756 
31. Hiraoka A, Kumada T, Michitaka K, Kudo M. Newly proposed ALBI grade and ALBI-T score as tools for assessment of hepatic function and prognosis in hepatocellular carcinoma patients. Liver Cancer. (2019) 8:31225. doi: 10.1159/000494844

32. Na SK, Yim SY, Suh SJ, Jung YK, Kim JH, Seo YS, et al. Versus Child-Pugh grading systems for liver function in patients with hepatocellular carcinoma. J Surg Oncol. (2018) 117:912-21. doi: 10.1002/jso. 24992

33. Yilmaz A, Mirili C, Bilici M. Tekin SB. A novel predictor in patients with gastrointestinal stromal tumors: Systemic immune-inflammation index (SII). J BUON. (2019) 24:2127-35.

34. Yan Q, Ertao Z, Zhimei Z, Weigang D, Jianjun P, Jianhui C, et al. Systemic immune-inflammation index (SII): a more promising inflammationbased prognostic marker for patients with synchronic colorectal peritoneal carcinomatosis. J Cancer. (2020) 11:5264-72. doi: 10.7150/jca. 46446

35. Ward E, Hopkins M, Arbuckle L, Williams N, Forsythe L, Bujkiewicz $\mathrm{S}$, et al. Nutritional problems in children treated for medulloblastoma: implications for enteral nutrition support. Pediatr Blood Cancer. (2009) 53:570-5. doi: 10.1002/pbc.22092

36. Yu X, Wen Y, Lin Y, Zhang X, Chen Y, Wang W, et al. The value of preoperative Glasgow prognostic score and the C-Reactive protein to albumin ratio as prognostic factors for long-term survival in pathological T1N0 esophageal squamous cell carcinoma. J Cancer. (2018) 9:80715. doi: $10.7150 /$ jca. 22755

Conflict of Interest: The authors declare that the research was conducted in the absence of any commercial or financial relationships that could be construed as a potential conflict of interest.

Publisher's Note: All claims expressed in this article are solely those of the authors and do not necessarily represent those of their affiliated organizations, or those of the publisher, the editors and the reviewers. Any product that may be evaluated in this article, or claim that may be made by its manufacturer, is not guaranteed or endorsed by the publisher.

Copyright (c) 2021 Zhu, Cheng, Hu, Chen, Zhang, Ke, Yang, Lin, Chen and Wang. This is an open-access article distributed under the terms of the Creative Commons Attribution License (CC BY). The use, distribution or reproduction in other forums is permitted, provided the original author(s) and the copyright owner(s) are credited and that the original publication in this journal is cited, in accordance with accepted academic practice. No use, distribution or reproduction is permitted which does not comply with these terms. 